\title{
Stimulus-Dependent Changes in Spike Threshold Enhance Feature Selectivity in Rat Barrel Cortex Neurons
}

\author{
W. Bryan Wilent and Diego Contreras \\ Department of Neuroscience, University of Pennsylvania School of Medicine, Philadelphia, Pennsylvania 19106
}

Feature selectivity is a fundamental property of sensory cortex neurons, yet the mechanisms underlying its genesis are not fully understood. Using intracellular recordings in vivo from layers 2-6 of rat barrel cortex, we studied the selectivity of neurons to the angular direction of whisker deflection. The spike output and the underlying synaptic response decreased exponentially in magnitude as the direction of deflection diverged from the preferred. However, the spike output was more sharply tuned for direction than the underlying synaptic response amplitude. This difference in selectivity was attributable to the rectification imposed by the spike threshold on the input- output function of cells. As in the visual system, spike threshold was not constant and showed trial-to-trial variability. However, here we show that the mean spike threshold was direction dependent and increased as the direction diverged from the preferred. Spike threshold was also related to the rate of rise of the synaptic response, which was direction dependent and steepest for the preferred direction. To assess the impact of the direction-dependent changes in spike threshold on direction selectivity, we applied a fixed threshold to the synaptic responses and calculated a predicted spike output. The predicted output was more broadly tuned than the obtained spike response, demonstrating for the first time that the regulation of the spike threshold by the properties of the synaptic response effectively enhances the selectivity of the spike output.

Key words: action potential; tuning; whisker; direction; synchronization; synaptic potential; intracellular; in vivo; feature selectivity

\section{Introduction}

Neurons in sensory cortices respond preferentially to certain attributes of stimuli within their specific sensory modality (i.e., they are tuned to specific stimulus features). Understanding the cellular and network mechanisms that underlie stimulus feature selectivity is an essential step toward understanding the strategies used by the neocortex for stimulus representation.

In primary visual cortex, for example, neurons are tuned to stimulus features such as orientation and spatial and temporal frequency (for review, see Ferster, 2004). Orientation tuning is believed to arise from the pattern of thalamocortical projections (Hubel and Wiesel, 1962; Alonso et al., 1996; Ferster, 2004), but the role of additional cortical mechanisms is still not fully understood (Chance et al., 1999; Martin, 2002). In the barrel cortex of rodents, neurons are selective for the angular direction in which their corresponding mystacial whisker [the principal whisker $(\mathrm{PW})$ ] is deflected (Simons, 1983; Simons and Carvell, 1989; Bruno and Simons, 2002). Direction selectivity is already present in the principal trigeminal nucleus (Minnery and Simons, 2003; Shoykhet et al., 2003) and in the ventrobasal nucleus of the thalamus (Simons and Carvell, 1989; Bruno et al., 2003; Timofeeva et al., 2003). Therefore, the spike output tuning of individual barrel

\footnotetext{
Received Sept. 22, 2004; revised Jan. 8, 2005; accepted Feb. 2, 2005.

This work was sponsored by The Human Frontier Science Program Organization. We thank Jessica Cardin, Gene Civillico, Leif Finkel, and Michael Higley for their helpful comments in preparing this manuscript and Esther Garcia de Yebenes for her technical assistance.

Correspondence should be addressed to Diego Contreras, Department of Neuroscience, University of Pennsylvania School of Medicine, 215 Stemmler Hall, Philadelphia, PA 19106-6074. E-mail: diego@@mail.med.upenn.edu. D0I:10.1523/JNEUROSCI.4906-04.2005

Copyright $\odot 2005$ Society for Neuroscience $\quad$ 0270-6474/05/252983-09\$15.00/0
}

cortex cells may simply be a faithful reproduction of the tuning properties of their synaptic inputs, or, alternatively, postsynaptic mechanisms may transform a broadly tuned synaptic input into a sharply tuned spike output. The latter imposes less stringent anatomical requirements on the system.

A postsynaptic mechanism for enhancing stimulus selectivity in primary visual cortex is the spike threshold (Carandini and Ferster, 2000; Volgushev et al., 2000, 2002). The spike output is more sharply tuned than the synaptic responses, because nonoptimal stimuli evoke primarily subthreshold responses. In addition, it was proposed that an adaptive threshold, regulated by the rate of rise of the synaptic response, may further enhance stimulus selectivity by decreasing the threshold, and therefore increasing the output, to optimal stimuli (Bonds, 1989; Nelson et al., 1994; Azouz and Gray, 2003). Such a mechanism has never been demonstrated but has strong support from experimental studies showing that spike threshold depends on the rate of rise of the triggering membrane potential $\left(V_{\mathrm{m}}\right)$ trajectory (Hodgkin and Huxley, 1952; Noble and Stein, 1966; Schlue et al., 1974; Bryant and Segundo, 1976; Fricker et al., 1999; Azouz and Gray, 2000). In contrast, it has been shown experimentally that stimuli of optimal orientation have steeper input-output relationships, but this is not because of the spike threshold, which does not change with orientation (Volgushev et al., 2002).

Here, we show for the first time that stimulus-dependent changes in spike threshold play a key role in sharpening the tuning to a stimulus feature (i.e., the angular direction of whisker deflection in rat barrel cortex neurons). The spike threshold is direction dependent and related to the slope of the synaptic response. The systematic increase in spike threshold as the direc- 
tion deviates from the preferred effectively rendered more nonpreferred trials subthreshold and enhanced the selectivity of the spike output.

\section{Materials and Methods}

Surgery and preparation. Experiments were conducted in accordance with the ethical guidelines of the National Institutes of Health and with the approval of the Institutional Animal Care and Use Committee of the University of Pennsylvania (Philadelphia, PA). Adult male Sprague Dawley rats $(350-450 \mathrm{~g})$ were anesthetized with pentobarbital $(50 \mathrm{mg} / \mathrm{kg}$, i.p.). Buprenorphine $(0.03 \mathrm{mg} / \mathrm{kg}$, s.c.) was administered to provide additional analgesia. Animals were paralyzed with gallamine triethiodide and artificially ventilated. End-tidal $\mathrm{CO}_{2}(3.5-3.7 \%)$ and heart rate were continuously monitored. Body temperature was maintained at $37^{\circ} \mathrm{C}$ via servo-controlled heating blanket and rectal thermometer (Harvard Apparatus, Holliston, MA). The depth of anesthesia was maintained by supplemental doses of the same anesthetic to keep a constant heart rate and a constant high-amplitude, low-frequency electroencephalogram as recorded from a bipolar electrode inserted into the cortex.

For cortical intracellular recordings, the animal was placed in a stereotaxic apparatus (David Kopf Instruments, Tujunga, CA), and a craniotomy was made to expose the surface of the barrel cortex $(1.0-3.0 \mathrm{~mm}$ posterior to bregma, $4.0-7.0 \mathrm{~mm}$ lateral to the midline). The dura was resected over the recording area, and mineral oil was applied to prevent desiccation. The stability of recordings was improved by drainage of the cisterna magna, hip suspension, and filling of the holes made for recording with a solution of $4 \%$ agar.

Electrophysiological recordings. Intracellular recordings were performed with glass micropipettes filled with $3 \mathrm{~m}$ potassium acetate and DC resistances of 80-90 $\mathrm{M} \Omega$. The intracellular recordings were usually obtained within $1 \mathrm{~mm}$ of the EEG recording electrode. Pipettes were oriented normal to the cortical surface, and the vertical depth was read on the scale of the micromanipulator. A high-impedance amplifier (bandpass, $0-5 \mathrm{kHz}$ ) with active bridge circuitry (Cygnus Technology, Delaware Water Gap, PA) was used to record and inject current into the cells. Data were digitized at $10 \mathrm{kHz}$ and stored on Nicolet Vision (Nicolet Instrument Technologies, Madison, WI). A computer operating LabView (National Instruments, Austin, TX) was used for the on-line averaging of responses.

Whisker stimulation. Before recording, whiskers were trimmed to a length of $\sim 12 \mathrm{~mm}$. Individual whiskers were deflected mechanically using ceramic piezoelectric bimorphs (Simons, 1983; Wilent and Contreras, 2004). Once a stable recording was obtained, the cell was depolarized moderately to facilitate whisker-evoked suprathreshold responses. Given the extensive subthreshold receptive fields of barrel cortex neurons (Moore and Nelson, 1998; Zhu and Connors, 1999; Brecht and Sakmann, 2002; Brecht et al., 2003; Higley and Contreras, 2003), a minimum of six whiskers was tested for each cell. The whisker that evoked the most spikes was deemed the PW. The PW was then repeatedly deflected in eight randomly interleaved directions, separated by $45^{\circ}$, using a velocity of $1300^{\circ} / \mathrm{s}$ (acceleration, $7.8 \times 10^{5 \%} / \mathrm{s}^{2}$ ). Caudal deflections are referred to as the $0^{\circ}$ direction, rostral deflections as $180^{\circ}$, dorsal deflections as $90^{\circ}$, and ventral deflections as $270^{\circ}$ (see Fig. $1 \mathrm{~A}$ ). The direction that evoked the most spikes was deemed the preferred direction (PD). All stimuli were $100 \mathrm{~ms}$ ramp, and hold deflections were delivered at $0.5 \mathrm{~Hz}$ or less to prevent steady-state adaptation of whisker-evoked responses (Moore et al., 1999). For trials at depolarized and hyperpolarized $V_{\mathrm{m}} \mathrm{s}$, current pulses were applied at least $200 \mathrm{~ms}$ before whisker stimuli to ensure the $V_{\mathrm{m}}$ had reached steady state and to avoid capacitive artifacts. The amount of current injected was adjusted for each cell depending on its input resistance and firing rate at depolarized potentials.

Data analysis. All data analyses were performed off-line. Routines for averaging sensory responses were written in Igor Pro (WaveMetrics, Lake Oswego, OR). For all cells, whisker-evoked postsynaptic potential amplitudes were measured from the baseline $V_{\mathrm{m}}$ to the peak of the response. Baseline $V_{\mathrm{m}}$ was calculated as the mean $V_{\mathrm{m}}$ of the $100 \mathrm{~ms}$ preceding whisker deflection. The direction sensitivity index (DI) of cells was measured as the PD response divided by the average response of all directions
(Bruno and Simons, 2002). The DI of spikes was measured using total evoked spikes, and the DI of the $V_{\mathrm{m}}$ was measured using the synaptic response amplitude. Spike threshold was measured at the peak of the second derivative of the $V_{\mathrm{m}}$ trace of the synaptic response, because threshold represents the highest acceleration of the membrane depolarization around a spike, which occurs when the membrane capacitance is discharged as the ionic current switches from outward to inward. This defines the action potential threshold (Noble, 1966). Spike threshold was measured relative to the baseline $V_{\mathrm{m}}$ immediately preceding the response. This procedure was checked repeatedly by visual inspection to assure its robustness; in all cases, the second derivative provided an accurate and reliable detection of the spike threshold. Cells $(n=8)$ in which the action potential threshold could not be delineated unambiguously were not included in the database. The time window of $2.5 \mathrm{~ms}$ preceding threshold to measure the $d V_{\mathrm{m}} / d t$ was chosen because a spike response was often evoked within 3-4 ms of response onset; therefore, using a range larger than $2.5 \mathrm{~ms}$ would potentially include time points at which the $V_{\mathrm{m}}$ was almost certainly not changing as a result of the stimulus. In rare instances in which a spike was evoked within $2.5 \mathrm{~ms}$ of response onset, $d V_{\mathrm{m}} / d t$ was determined over the appropriately shortened range (response onset to spike onset). However, this is not the preferred method for the population because of the difficulty in precisely determining stimulus onset.

Statistical measures were calculated using Igor Pro. An ANOVA was calculated to compare more than two groups, and Tukey's honestly significantly different post hoc test was used for pairwise comparisons. When comparing just two groups, Student's $t$ test was used.

\section{Results}

We recorded intracellularly in vivo from neurons in layers 2-6 of the rat barrel cortex. All cells included in the database $(n=32)$ had a stable $V_{\mathrm{m}}$ between -66 and $-79 \mathrm{mV}(-72 \pm 5 \mathrm{mV}$; mean $\pm \mathrm{SE}$ ) for at least $40 \mathrm{~min}$, overshooting action potentials, and were sensitive to the direction of whisker deflection. Cells with direction indices (see below) lower than 1.3 were not included in the database $(n=12)$. In addition, fast-spiking cells $(n=3)$, as determined by their firing pattern in response to intracellular current injection (Connors and Gutnick, 1990; Nunez et al., 1993), were not included, because this population is insensitive to the direction of whisker deflection (Bruno and Simons, 2002). The average action potential duration of cells included in the database was $1.0 \mathrm{~ms}$ (range, $0.8-1.2 \mathrm{~ms}$ ). We will present the results as follows: first, we will compare the direction sensitivity and the direction tuning of the spike output to the underlying synaptic response amplitude. Second, we will show that the rate of rise $\left(d V_{\mathrm{m}} / d t\right)$ of the synaptic response and the spike threshold are direction dependent and correlated. Third, we will show that the variation of spike threshold with direction effectively sharpens direction tuning.

\section{Direction sensitivity and direction tuning of rat barrel cortex neurons}

The PW was determined for each cell (see Materials and Methods) and was then deflected repeatedly $(n=15-20)$ in eight randomly interleaved directions (Fig. $1 A$ ). The direction that evoked the most spikes was deemed the PD. For the example cell shown in Figure 1, deflections in the caudorostral direction $\left(135^{\circ}\right)$ evoked the most spikes, as shown in the peristimulus spike histogram (11 spikes) (Fig. $1 B$ ). The synaptic response evoked by that direction was also the largest in amplitude, measured as the change in voltage from baseline to the peak of the response after spike removal $(7.4 \mathrm{mV})$ (Fig. 1C). The direction sensitivity of barrel cortex neurons to whisker deflection is usually quantified using the DI measure (Bruno and Simons, 2002), which is the number of spikes evoked by the PD divided by the average num- 

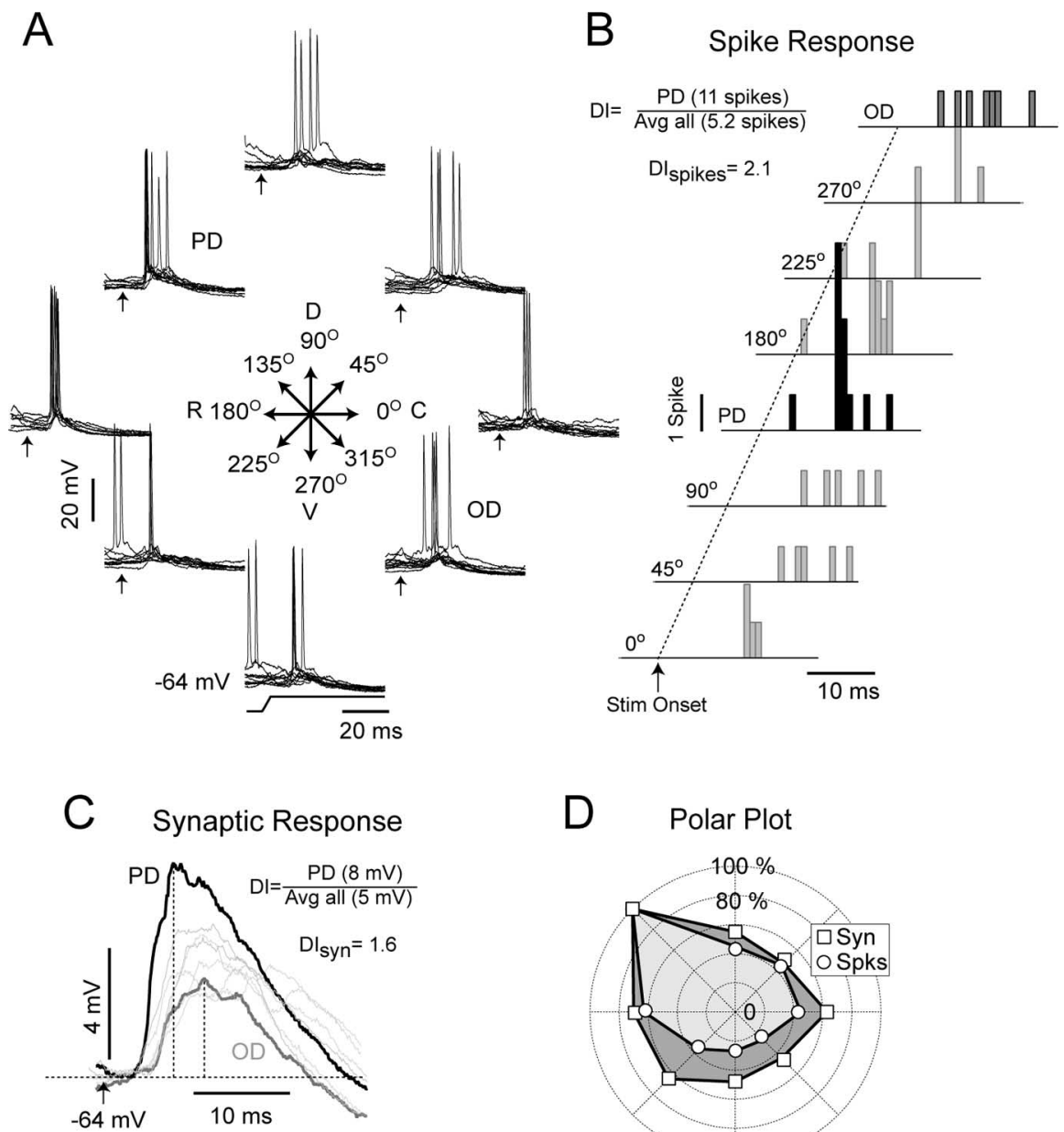

E

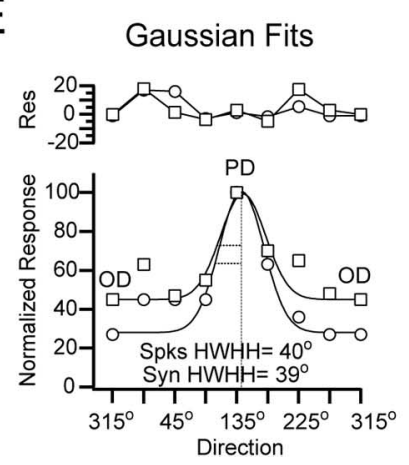

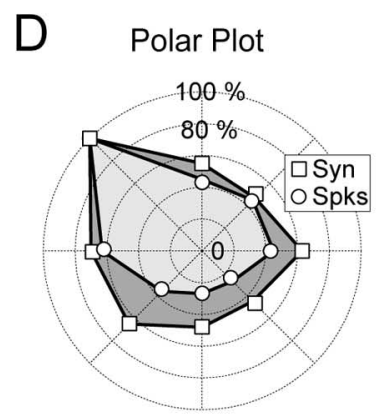

F Exponential Fits

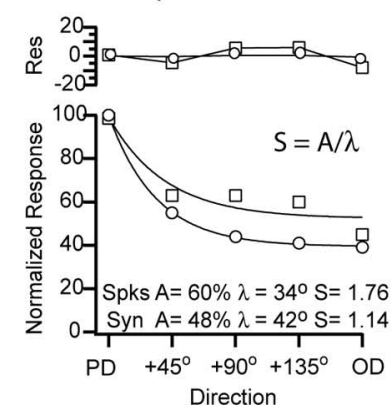

Figure 1. Synaptic and spike responses to different directions of whisker deflection. $\boldsymbol{A}$, Superimposed single trial responses to eight directions ( $45^{\circ}$ intervals) obtained at a moderately depolarized $V_{m}(-64 \mathrm{mV} ; 0.2 \mathrm{nA})$ are shown. The dorsorostral direction $\left(135^{\circ}\right)$, which evoked 11 spikes, was the $P D$, and the $0 D, 315^{\circ}$, evoked four spikes. Arrows indicate stimulus onset. D, Dorsal; $V$, ventral; $R$, rostral; $C$, caudal. $\boldsymbol{B}$, Poststimulus spike histograms of the responses to all directions (indicated at right), offset horizontally and vertically for clarity, are shown. The dotted line indicates time of onset of whisker deflection (Stim Onset). The DI for this cell was 2.1. Avg, Average. C, Superimposed average $(n=17)$ synaptic responses are shown. Vertical dotted lines indicate the peak where the amplitude was measured. The DI for the synaptic response ( $\mathrm{DI}_{\text {syn }}$ ) amplitude was 1.6. Response to the PD (black trace) and the OD (gray trace) are represented as thicker lines to highlight their differences. Stimulus onset time is indicated by the arrow. D, A polar plot of the spike (Spks; circle) and synaptic (Syn; square) response magnitude is shown. The spike response was more sharply tuned. $\boldsymbol{E}$, The bottom plot shows the response magnitude plotted versus direction fit by a Gaussian function. The HWHH of the fit did not distinguish between the spike and synaptic response amplitude. The top plot shows the residuals (Res) from the fit. $\boldsymbol{F}$, Response magnitude, plotted as function of angular distance from the PD, was fit with an exponential function, and the decay constant and decay amplitude of the fit were used to determine the selectivity. The spike response was more selective than the synaptic response amplitude. The top plot shows the residuals from the fit.

ber of spikes evoked by all directions. For this cell, the DI of the spike response $\left(\mathrm{DI}_{\text {spikes }}\right)$ was 2.1. We also calculated the DI of the synaptic response amplitude $\left(\mathrm{DI}_{\mathrm{syn}}=1.6\right)$ by substituting millivolts for spikes. Thus, according to the DI measure, the spike output of the cell was more sensitive to direction than the underlying synaptic response amplitude.

The DI, however, does not provide information about direction tuning, because it only indicates the strength of the $\mathrm{PD}$ response relative to the average response from all directions. The tuning for direction can be assessed qualitatively by plotting response magnitude in polar coordinates (Fig. 1D). For this cell, the differences in spike counts were larger among directions than the differences in synaptic response amplitude; thus, qualitatively, the spike output was tuned more sharply than the synaptic response amplitude. We fit the data with a Gaussian function to quantify the tuning, but this approach yielded unsatisfactory fits (Fig. $1 E$ ). The residual variance was high (spike response, 5.8\%; synaptic response, $6.4 \%$ ) (Fig. $1 E$, top plot, residuals), and the halfwidth at half-height (HWHH) of the Gaussian failed to detect the difference in tuning between the spike output and the synaptic response ( $\mathrm{HWHH}$ for spikes, $76^{\circ}$; for the synaptic response, $77^{\circ}$ ) (Fig. $1 E$ ) that was clear in the polar plot.

To obtain a sensitive and reliable measure of direction tuning, we collapsed the measures from directions equidistant from the $\mathrm{PD}$; consequently, response magnitude values were obtained for five directions: PD, $45^{\circ}$ away from PD (average, 45 and $315^{\circ}$ ), $90^{\circ}$ away (average, 90 and $270^{\circ}$ ), $135^{\circ}$ away (average, 135 and $225^{\circ}$ ), and $180^{\circ}$ away, which is the opposite direction (OD). To quantify direction tuning, the response magnitudes were fit with an exponential function, which yielded less residual variance than the Gaussian fits (spike response, $0.4 \%$; synaptic response, $4.8 \%$ ) (Fig. $1 F$ ). From the parameter values of the exponential fits, we generated a selectivity index $(S)$, calculated as the ratio between the amplitude of the decay $(A)$ from the PD to the OD and the decay constant $(\lambda)$. The values of $S$ obtained from the exponential fits reflected the difference in tuning evident in the polar plots (synaptic: $\lambda=42^{\circ}, A=48 \%, S=$ 1.14; spikes: $\lambda=34^{\circ} ; A=60 \%, S=1.76$ ) (Fig. $1 E$ ). In summary, the spike response was more sensitive (larger DI) and more selective (larger $S$ ) for direction than the amplitude of the underlying synaptic response. 
We compared the direction sensitivity and the direction selectivity between the spike and the synaptic responses for all cells. Similar to what is reported in other intracellular studies performed in this system (Moore and Nelson, 1998; Brecht and Sakmann, 2002; Higley and Contreras, 2003; Wilent and Contreras, 2004), a small number of spikes per stimulus ( 0.28 spikes per stimulus) was evoked by the $\mathrm{PD}$ at the resting $V_{\mathrm{m}}(-72 \pm 5 \mathrm{mV})$. Therefore, we measured the spike responses while cells were slightly depolarized (7-12 $\mathrm{mV}$ above rest; average $\left.V_{\mathrm{m}}=-63 \pm 3 \mathrm{mV}\right)$ with square current pulses $(+0.1$ to $+0.3 \mathrm{nA}$ ) of $0.6 \mathrm{~s}$ duration and starting $0.2 \mathrm{~s}$ before each whisker deflection. This procedure increased the average spike output to 0.88 spikes per stimulus for the PD, which is more comparable with what is reported in extracellular studies (Simons and Carvell, 1989). The disparity in activity values reported in the literature may be attributable to differences in anesthesia or a selection bias caused by the type of electrode or recording method. In addition to measuring the synaptic response amplitude at the depolarized level, we also measured it at rest and at a hyperpolarized $V_{\mathrm{m}}(-81 \pm 4$ $\mathrm{mV})$. The spike response was more sensitive to direction ( $\mathrm{DI}=2.12$ ) than the synaptic response amplitude at any of the three $V_{\mathrm{m}} \mathrm{s}$ (depolarized, DI $=1.78$; at rest, $\mathrm{DI}=1.42$; hyperpolarized, DI $=1.45 ; p<$ 0.01; one-way ANOVA) (Fig. $2 A$ ). In addition, the synaptic response amplitude at a depolarized $V_{\mathrm{m}}(-63 \mathrm{mV}$; DI $=1.78)$ was significantly more sensitive to direction than the response at rest $(-72 \mathrm{mV}$; $\mathrm{DI}=1.42 ; p<0.05)$ or at a hyperpolarized $V_{\mathrm{m}}(-81 \mathrm{mV}$; DI $=1.45 ; p<0.05)$ (Fig. $2 A)$. For all but one cell, the spike response was more sensitive to direction than the synaptic response amplitude measured at rest (Fig. 2 B). The range of DI values for the spike response (1.4-3.4) was larger than the synaptic response amplitude at rest (1.2-2.1) (Fig. 2C), and its distribution peak was shifted to the right.

To compare the direction selectivity of the spike output and the synaptic response amplitude for the whole population, we averaged the responses from all cells and generated population polar plots. To average between cells, the PD was plotted at $180^{\circ}$, and the response of each cell was normalized to its PD. It is clear from the population polar plots that the tuning of the spike output was sharper than the tuning of the synaptic response amplitude (Fig. $3 A$ ). To obtain the direction selectivity for the population (as shown for a single cell in Fig. 1), the data were plotted as a function of angular distance from the PD and fit with an exponential function. The residual variance of the fits was small (spikes, $2.2 \%$; depolarized, $1.8 \%$; at rest, $0.7 \%$; hyperpolarized, $0.5 \%$ ), as were the residuals, which are shown above the data (Fig. $3 B$ ). The selectivity of the

A
B
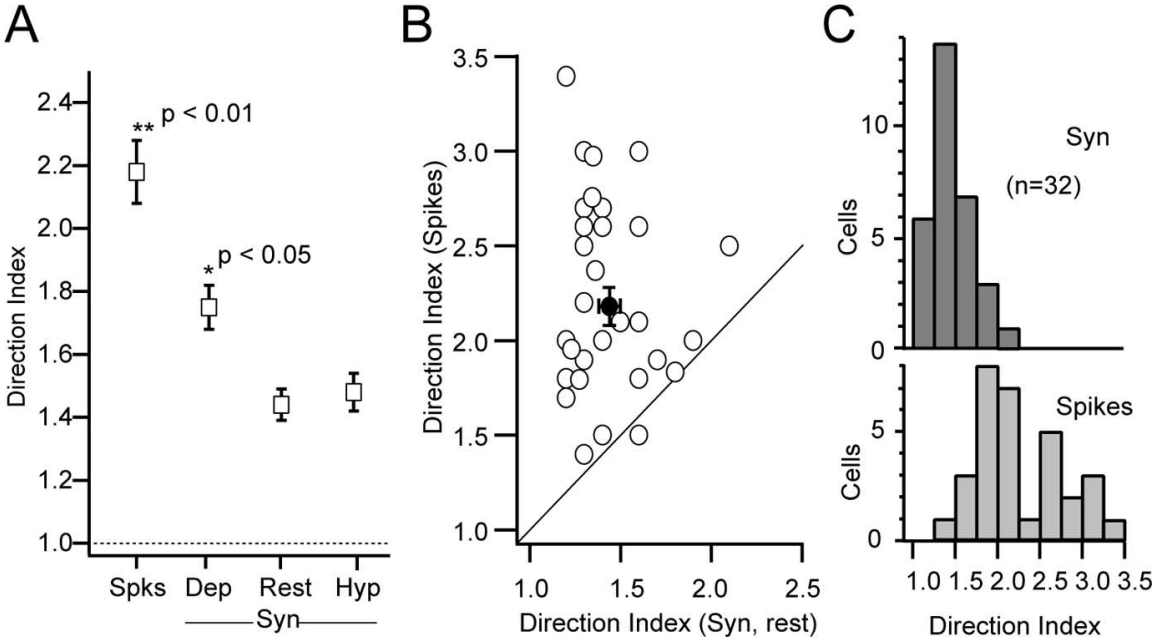

Figure 2. Direction sensitivity of spike and synaptic responses for the population. $\boldsymbol{A}$, The mean spike response (Spks; $\mathrm{DI}=2.18)$ was significantly $\left({ }^{* *} p<0.01\right)$ more sensitive to the direction of whisker deflection than the mean synaptic response (Syn) amplitude. The mean synaptic response amplitude under depolarization (Dep; $V_{m}=-63 \mathrm{mV}$; / range, $0.1-0.3 \mathrm{nA} ; \mathrm{DI}=1.78$ ) was significantly $\left({ }^{*} p<0.05\right)$ more sensitive than the mean response at rest $\left(V_{m}=-72 \mathrm{mV} ; \mathrm{DI}=1.42\right)$ or under hyperpolarization (Hyp; $V_{m}=-81 \mathrm{mV}$;/ range, $-0.2-0.5 \mathrm{nA} ; \mathrm{DI}=1.47$ ). Values are mean $\pm \mathrm{SE}$. $\boldsymbol{B}$, A plot of $\mathrm{DI}$ of the spike response versus the synaptic response at rest for each cell shows that all points except one are above the main diagonal. The filled circle indicates the average DI of the spikes and synaptic response. C, A histogram of the distribution of direction indices for the spike and synaptic

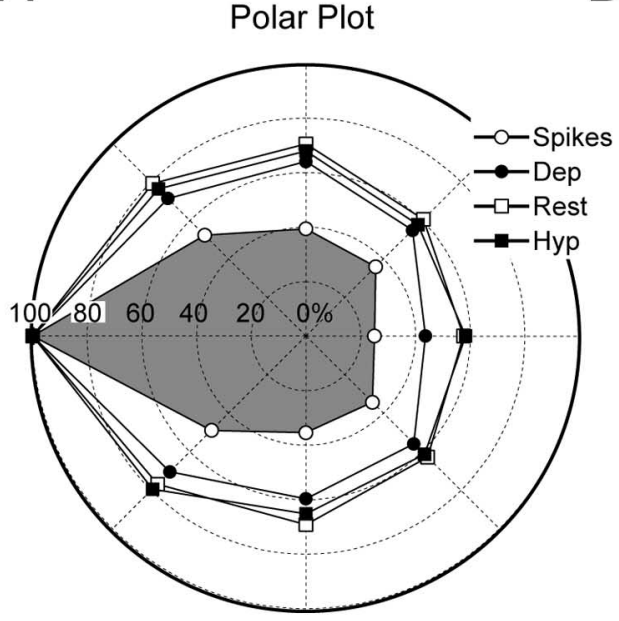

B

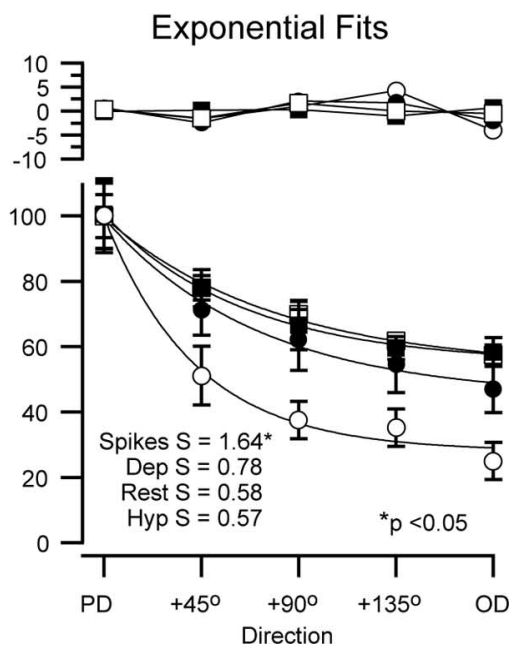

Figure 3. Direction tuning of the whole population. $A$, A population polar plot of the spike and synaptic responses, at depolarized (Dep), resting (Rest), and hyperpolarized (Hyp) $V_{m} s$, normalized to the $P D$, is shown. Polar plots artificially aligned to $180^{\circ}$. $\boldsymbol{B}$, Exponential fits of the population data plotted as function of distance from the PD are shown. Values are mean \pm SE. The selectivity of the spike response $(S=1.64)$ was significantly higher $(p<0.05)$ than the synaptic response amplitude (Dep, $S=$ 0.78 ; rest, $S=0.58 ;$ Hyp, $S=0.57$ ). Residuals are shown in the top plot.

population spike response $(S=1.64)$ was significantly higher than the selectivity of the population synaptic response amplitude, which was highest at the depolarized $V_{\mathrm{m}}$ (depolarized, $S=$ 0.78 ; at rest, $S=0.58$; hyperpolarized, $S=0.57$; all comparisons had $p<0.05$ ) (Fig. 3B). Hyperpolarization decreased selectivity and increased the amplitude of the synaptic responses; this change in amplitude was expected as a result of the increase in driving force of excitatory currents and possibly reversal of inhibitory currents but was in contrast to the decrease in amplitude with hyperpolarization reported during stimulation in the visual system (Azouz and Gray, 2003). To verify the accuracy of the tuning values obtained from the population polar plots, we ob- 


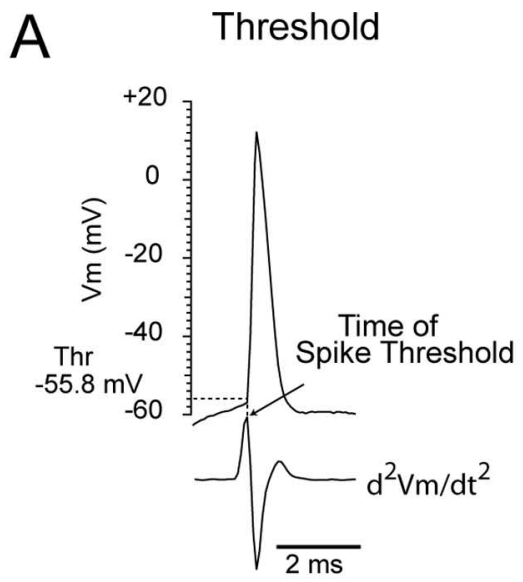

C PD vs OD Evoked Spikes

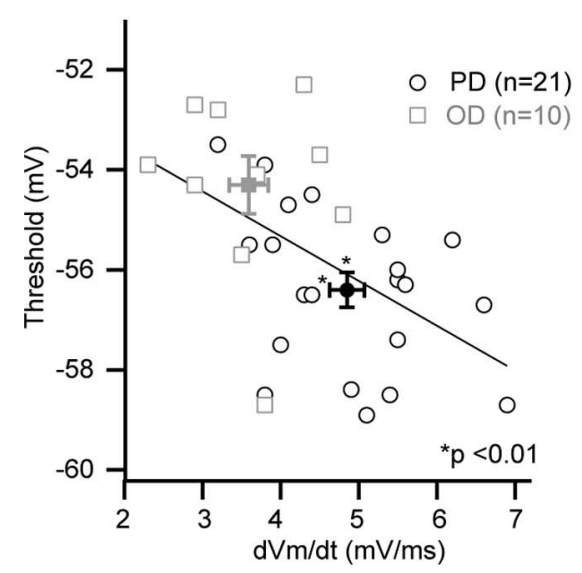

B PD vs OD Spikes

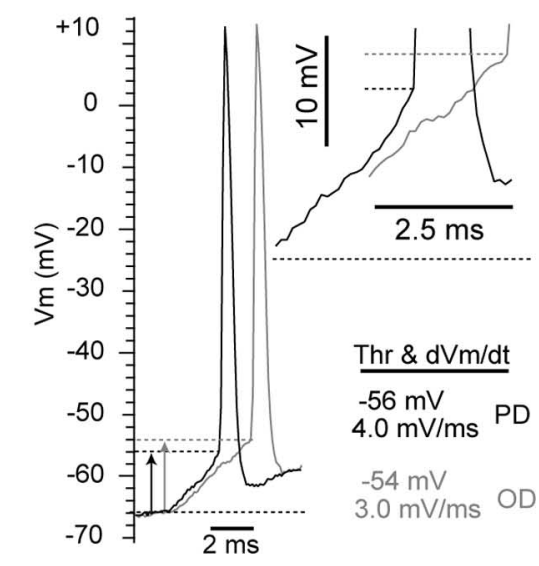

D Spontaneous Spikes

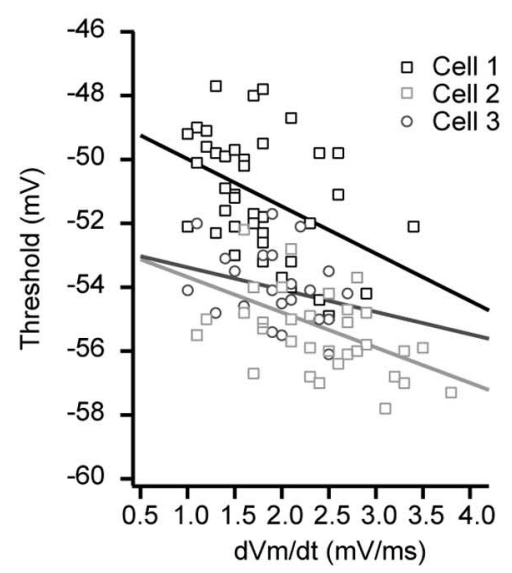

Figure 4. Spike threshold and the preceding $d V_{\mathrm{m}} / d t$. $\boldsymbol{A}$, The spike threshold (Thr) was determined as the $V_{\mathrm{m}}$ from baseline at the time of the peak of the second derivative. $B, A P D$ - (black) and OD- (gray) evoked spike are shown. The rate of rise preceding the $\mathrm{PD}$ spike was $1 \mathrm{mV} / \mathrm{ms}$ greater than that of the OD-evoked spike, and the spike threshold of the PD was $2.0 \mathrm{mV}$ lower than that of the OD-evoked spike (vertical arrows). The inset shows the final $2.5 \mathrm{~ms}$ of the synaptic response leading to a spike, the time period in which $d V_{\mathrm{m}} / d t$ was determined. $C$, Threshold and $d V_{\mathrm{m}} / d t$ of all OD- and PD-evoked spikes are shown. The spike threshold of PD-evoked spikes was significantly lower, and the $d V_{m} / d t$ was significantly higher than that of OD-evoked spikes (mean $\pm \mathrm{SE}$ ). D, Threshold versus $d V_{\mathrm{m}} / d t$ for spontaneous spikes from three example cells, including the cell shown in $\boldsymbol{B}$ (cell 1).

tained selectivity values for each individual cell, and their mean was similar to that of the population (spikes, $S=1.65 ; V_{\mathrm{m}}$ (rest), $S=0.68$ ). In summary, the direction selectivity of the spike response was higher than that of the underlying synaptic response amplitude, which was slightly higher when measured from a depolarized baseline $V_{\mathrm{m}}$.

\section{The relationship between spike threshold, $d V_{\mathrm{m}} / d t$, and direction}

The difference in selectivity between the synaptic and the spike responses demonstrated here was attributable in part to the nonlinearity introduced by the spike threshold, as already shown in other sensory systems (Azouz and Gray, 2000; Carandini and Ferster, 2000; Volgushev et al., 2000). However, if threshold changes with direction, it may serve to further enhance the selectivity of the spike output. Threshold is highly sensitive to the rate of rise of the $V_{\mathrm{m}}$ immediately preceding it (Hodgkin and Huxley, 1952; Schlue et al., 1974; Bryant and Segundo, 1976; Fricker et al., 1999; Henze and Buzsaki, 2001), and a cursory examination of the synaptic responses strongly suggested that the $d V_{\mathrm{m}} / d t$ was changing with direction. We measured spike threshold at the time of the peak of the second derivative of the $V_{\mathrm{m}}$ (Fig. $4 A$ ), and we calculated the $d V_{\mathrm{m}} / d t$ as the mean rate of rise during the $2.5 \mathrm{~ms}$ preceding spike initiation (see Materials and Methods). Representative responses to the PD and OD trial are shown for an example cell (Fig. $4 B$ ). The threshold of the PD trial was -56 or $10 \mathrm{mV}$ from baseline $(-66$ $\mathrm{mV}$ ), and the $d V_{\mathrm{m}} / d t$ of the response over the $2.5 \mathrm{~ms}$ preceding the spike was 4.0 $\mathrm{mV} / \mathrm{ms}$ (Fig. $4 B$ ). The threshold of the OD trial was -54 or $12 \mathrm{mV}$ from baseline, and the $d V_{\mathrm{m}} / d t$ of the response over the $2.5 \mathrm{~ms}$ preceding the spike was $3.0 \mathrm{mV} / \mathrm{ms}$ (Fig. $4 B$ ). For this cell, the mean spike threshold was significantly different between the two directions $(\mathrm{PD}=-56.4 \pm 0.4$ or $8.9 \pm 0.7 \mathrm{mV}$ above baseline; OD = $-54.3 \pm 0.6$ or $10.8 \pm 0.8 \mathrm{mV}$ above baseline; $p<0.01$ ) (Fig. $4 C$ ) as was the $d V_{\mathrm{m}} / d t$ $(\mathrm{PD}=4.8 \pm 0.2 \mathrm{mV} / \mathrm{ms} ; \mathrm{OD}=3.6 \pm 0.3$ $\mathrm{mV} / \mathrm{ms} ; p<0.01$ ) (Fig. $4 C$ ). An increase in the preceding $d V_{\mathrm{m}} / d t$ correlated with a decrease in spike threshold $(r=0.52 ; p<$ $0.02)$. The relationship between $d V_{\mathrm{m}} / d t$ and threshold was also verified for the spontaneous spikes from this cell $(r=$ $0.49 ; p<0.05$ ) (Fig. $4 D$, cell 1 ) as well as other cells (cell 2, $r=0.43, p<0.05$; cell 3, $r=0.34 ; p<0.05$ ) (Fig. 4D).

For the population, the $d V_{\mathrm{m}} / d t$ preceding a spike was distributed normally with a mean of $2.1 \pm 0.4 \mathrm{mV} / \mathrm{ms}$ (Fig. $5 A$ ). Stimulus-independent factors, such as spontaneous fluctuations in the baseline $V_{\mathrm{m}}$ that occur from trial to trial, can affect spike threshold (Azouz and Gray, 2003); therefore, we verified that the average baseline $V_{\mathrm{m}}$ was similar across directions for each cell. In addition, the level of current injection was adjusted for each cell such that the baseline depolarized $V_{\mathrm{m}}$ (range, -60 to $-66 \mathrm{mV}$ ) and the amount of depolarization required to reach threshold (range, 6-11 mV) were comparable for all cells. As the direction of deflection deviated from the $\mathrm{PD}$, spike threshold increased $(r=0.99 ; p<0.01)$ (Fig. $5 B)$. The amount of depolarization required to reach threshold for $\mathrm{PD}$-evoked spikes was significantly less $(8.0 \pm 0.18 \mathrm{mV} ; p<0.05)$ than the amount of depolarization required to reach threshold for spikes evoked from directions 90,135 , and $180^{\circ}$ away from the $\mathrm{PD}(9.0 \pm 0.18,9.1 \pm$ 0.22 , and $9.4 \pm 0.29 \mathrm{mV}$, respectively) (Fig. $5 B$ ). Accordingly, the $d V_{\mathrm{m}} / d t$ of the evoked spikes decreased with direction $(r=-0.95$; $p<0.02$ ) (Fig. 5C). The $d V_{\mathrm{m}} / d t$ of PD suprathreshold responses $(2.5 \pm 0.15 \mathrm{mV} / \mathrm{ms})$ was significantly higher than the $d V_{\mathrm{m}} / d t$ of responses evoked from directions 90,135 , and $180^{\circ}$ away from the $\mathrm{PD}(1.94 \pm 0.06,1.80 \pm 0.06$, and $1.79 \pm 0.12 \mathrm{mV} / \mathrm{ms}$, respectively) (Fig. $5 C$ ). Therefore, as predicted by the HodgkinHuxley model, as the $d V_{\mathrm{m}} / d t$ of the response decreased, the amount of depolarization required to reach threshold increased $(r=0.95 ; p<0.01)$ (Fig. $5 D)$. In summary, the $d V_{\mathrm{m}} / d t$ of the 

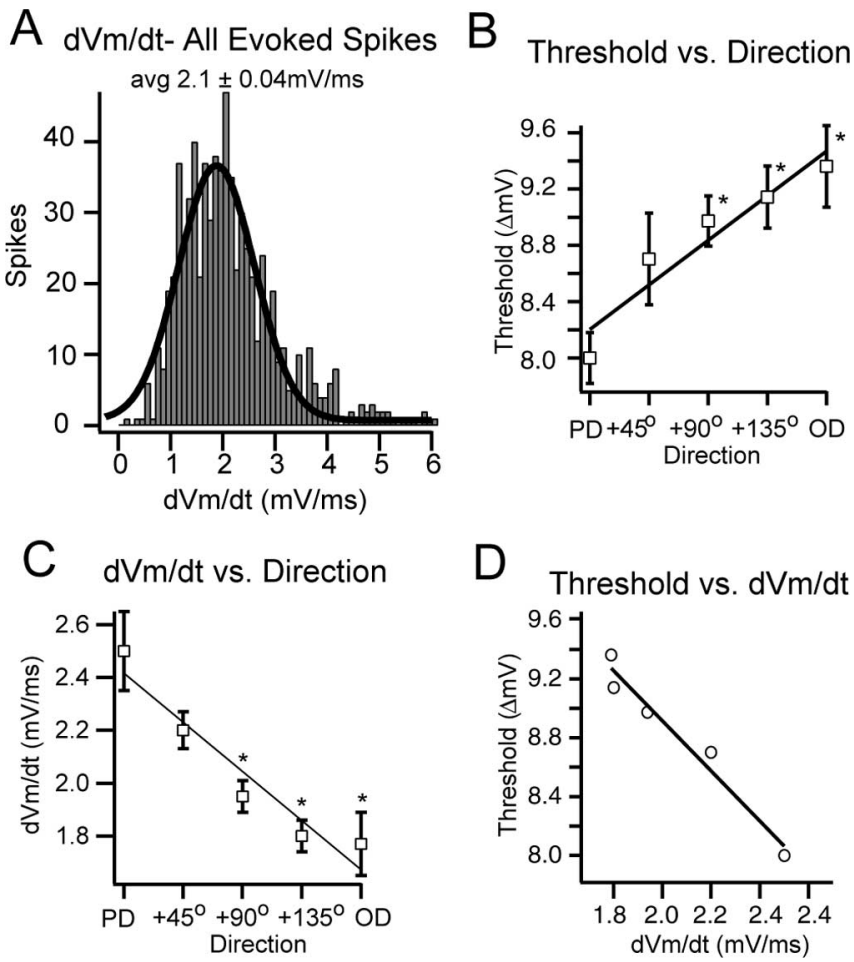

Figure 5. Relationship of spike threshold and $d V_{\mathrm{m}} / d t$ for the population. $\boldsymbol{A}$, The distribution of the $d V_{\mathrm{m}} / d t$ of all evoked spikes [mean (avg) $\pm \mathrm{SE}=2.1 \pm 0.04 \mathrm{mV} / \mathrm{ms}$ ] is shown. $\boldsymbol{B}$, The amount of depolarization required to reach spike threshold increased as the deflection direction moved away from PD. PD trials required significantly $\left({ }^{*} p<0.05\right)$ less depolarization than the $O D$ and directions 90 and $135^{\circ}$ away from the PD. C, The $d V_{m} / d t$ preceding a spike decreased as the direction deviated from the PD. The $d V_{\mathrm{m}} / d t$ preceding PD-evoked spikes $(2.5 \mathrm{mV} / \mathrm{ms})$ was significantly higher than the $d V_{m} / d t$ preceding spikes evoked by the $O D(1.78 \mathrm{mV} / \mathrm{ms})$ and directions $90^{\circ}(1.95 \mathrm{mV} / \mathrm{ms})$ and $135^{\circ}(1.80 \mathrm{mV} / \mathrm{ms})$ away from the PD. Error bars in $\boldsymbol{B}$ and $\boldsymbol{C}$ indicate SE. $D$, The relationship between spike threshold and $d V_{\mathrm{m}} / d t$ showed a negative correlation.

response and the spike threshold, which is directly regulated by the $d V_{\mathrm{m}} / d t$, are dependent on the direction of deflection.

\section{Contribution of spike threshold to direction tuning}

As the direction deviates from the preferred, an increase in spike threshold may render more trials subthreshold, because more depolarization is required to evoke a spike. This would effectively enhance selectivity by contributing to the difference in the spike output between preferred and nonpreferred directions. Because typically only one or two spikes were elicited by each deflection, it is not feasible to quantify spike output using models of spike generation, such as the rectification model (Granit et al., 1963; Movshon et al., 1978; Carandini and Ferster, 2000), which rely on mean firing rates. In addition, only the threshold of the first spike reliably reflects changes in the $d V_{\mathrm{m}} / d t$ of the synaptic response. The threshold of subsequent spikes are also affected by the interspike interval (Henze and Buzsaki, 2001). Therefore, to assess whether spike threshold contributes to direction selectivity, we compared the number of single trials that were suprathreshold for spike generation (suprathreshold/stimulus obtained) to the theoretical number of trials that would have been suprathreshold (suprathreshold/stimulus predicted) had the synaptic response generated a spike every time it crossed an arbitrary fixed threshold (Fig. 6). For each cell, the fixed threshold applied was the average threshold of spikes evoked by the PD. Therefore, the difference between the observed and the predicted number of suprathreshold trials per stimulus depends solely on the amplitude of the synaptic response. A difference between the obtained and the predicted value demonstrates that changes in spike threshold play a role in direction selectivity. In theory, the use of either the PD or OD average threshold produces the same result, namely, that the predicted number of suprathreshold trials differs from the obtained. Because the threshold varies among directions, any fixed threshold would reveal a difference between the predicted and the obtained output. The use of the OD selects fewer spikes for all directions, making comparisons less robust. In addition, our goal was to determine whether the increase in threshold for directions diverging from the PD enhanced selectivity; therefore, we applied the threshold of the PD.

The above measurement requires that direction selectivity be reflected primarily in the probability of suprathreshold trials and not in the number of spikes per suprathreshold trial. Therefore, we compared the selectivity index obtained with these two measures.

For the cell illustrated in Figure 6, the response to the PD was suprathreshold for spike generation in 16 of 21 trials (0.76 suprathreshold per stimulus, observed) (Fig. 6A, right). On two trials, a second spike was evoked generating 18 spikes in total (0.86 spikes per stimulus). The average spike threshold of PD evoked spikes was $-55.6 \mathrm{mV}$. In one trial, the amplitude of the synaptic response was more depolarized than the average spike threshold but did not generate a spike (Fig. 6A, inset, red trace); thus, the predicted number of suprathreshold trials using a fixed threshold $(-55.6 \mathrm{mV})$ was 17 (0.81 suprathreshold per stimulus, predicted). In the OD (Fig. $6 A$, left), 6 of 21 trials were suprathreshold (0.29 suprathreshold per stimulus, observed) with each trial generating a single spike ( 0.29 spikes per stimulus). Four synaptic responses crossed the fixed threshold value without generating spikes (red traces), and therefore, the predicted number of suprathreshold trials was 10 of 21 trials ( 0.48 suprathreshold per stimulus, predicted).

Thus, expressing the numbers as percentages, the number of spikes per stimulus evoked for the OD was $34 \%$ of that for the PD (Fig. $6 \mathrm{~B}$ ), and the number of suprathreshold trials per stimulus (observed) for the OD was only $38 \%$ of the PD, indicating that additional spikes per suprathreshold trial contributed little to the difference in output between the PD and OD. In contrast, the number of suprathreshold trials per stimulus for the OD was $58 \%$ of the PD (Fig. $6 \mathrm{~B}$ ) when predicted using a fixed threshold, indicating that the increase in threshold contributed to the difference between the number of suprathreshold trials between the PD and the OD (Fig. 6B). In summary, for this cell, the increase in threshold for the OD contributed to the selectivity of the spike output because, if threshold were fixed, the difference between the PD and OD would have been smaller.

We used this approach for the whole population and compared the tuning of the suprathreshold per stimulus obtained versus that predicted using a fixed threshold (Fig. 7). The difference was already clear in the polar plot (Fig. 7A). As in Figure 3, values were represented normalized to the PD and offset to $180^{\circ}$. The obtained values of the spike (gray circles) and synaptic (triangles) responses are those from the population shown in Figure 3 . The selectivity of the suprathreshold trials per stimulus obtained ( $S=1.55$ ) (Fig. $7 B$, open black circles) was significantly higher than that predicted by a fixed threshold $(S=0.98 ; p<$ 0.05 ) (Fig. $7 B$, filled black circles). In addition, the selectivity of the suprathreshold trials per stimulus obtained for the population was only slightly lower than the selectivity of the number of spikes per stimulus ( $S=1.64$ ), which indicates, as for the single 


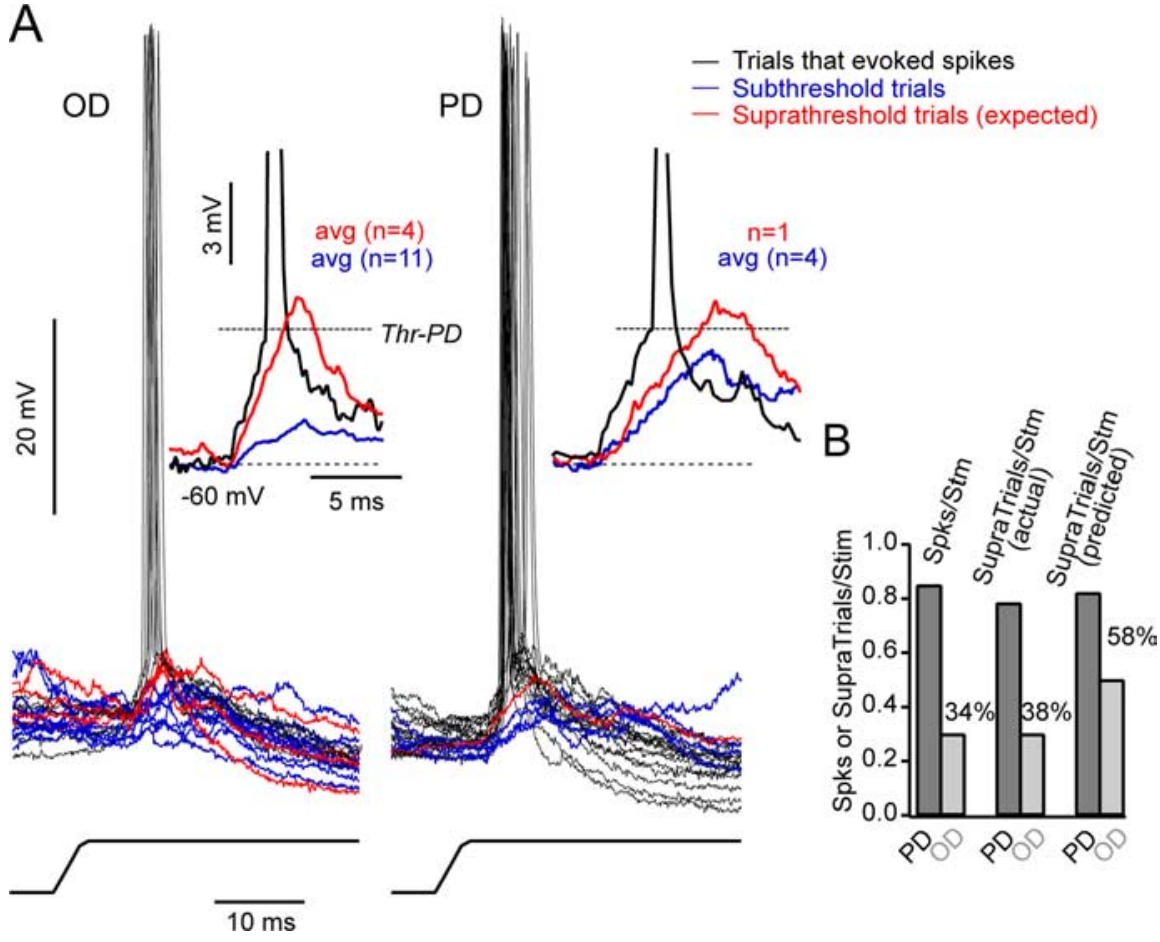

Figure 6. The effect of a varying spike threshold on the number of suprathreshold trials. $A$, At left, superimposed responses to the OD ( $n=21)$ showing suprathreshold trials that generated spikes ( $n=6$ trials, $n=6$ spikes; black), trials that crossed threshold (Thr) but did not generate spikes $(n=4)$, and subthreshold trials $(n=11)$. At right, superimposed responses to the PD $(n=21)$ showing suprathreshold trials that generated spikes $(n=16$ trials, $n=18$ spikes; black), trials that crossed threshold but did not generate spikes ( $n=1$; red), and subthreshold trials ( $n=4$; blue) [average (avg) $V_{\mathrm{m}}=-60 \mathrm{mV} ; I=0.2 \mathrm{nA}$ ]. Averages are shown in the insets. $\boldsymbol{B}$, The left histogram shows the spikes per stimulus (Spks/Stm) for the OD and the PD for this cell. The OD was $34 \%$ of the value of the PD. The middle histogram illustrates the number of trials per stimulus in which a spike was evoked (suprathreshold per stimulus observed). The $0 D$ was $35 \%$ of the PD, which is similar to the number of spikes per stimulus, because all but two suprathreshold trials evoked only a single spike. The right histogram shows the number of suprathreshold trials per stimulus predicted using a fixed threshold (this includes trials that crossed threshold but did not generate a spike; red traces in $\boldsymbol{A}$ ). The difference between directions is considerably smaller ( $O D=58 \%$ of $P D)$, showing that a variable threshold enhances direction selectivity.
A

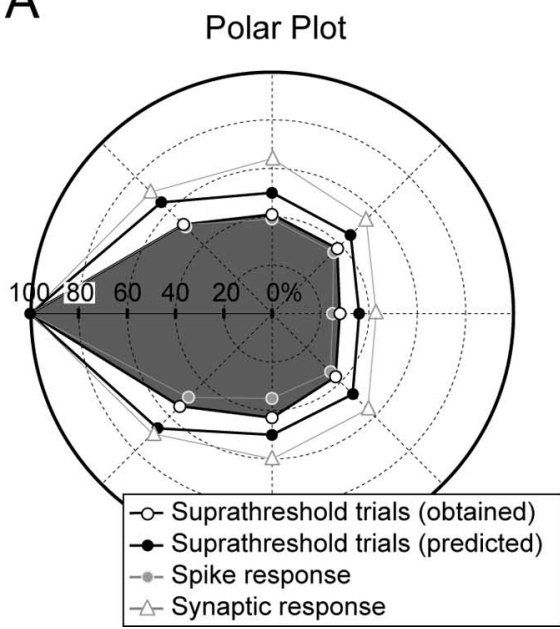

B

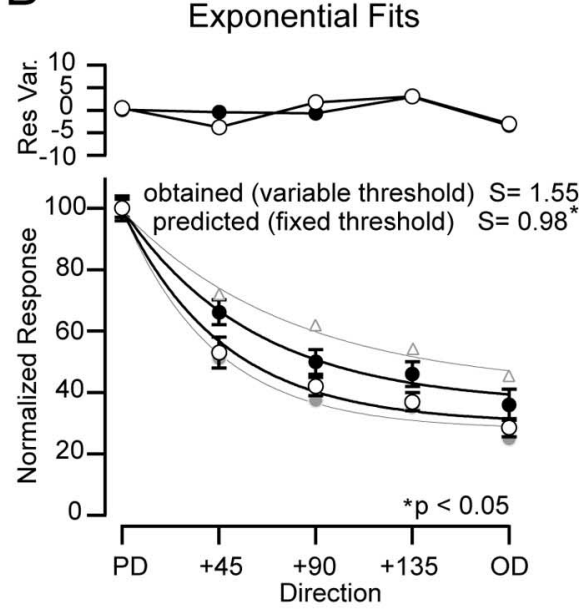

Figure 7. Comparison between obtained and predicted suprathreshold trials per stimulus for the population. A, Population polar plots showing the sharpening in tuning of the obtained suprathreshold trials (open black circles) compared with the number predicted using a fixed threshold (filled black circles). $\boldsymbol{B}$, Exponential fits to the obtained and predicted responses indicated that the selectivity of the obtained responses $(S=1.55)$ was significantly higher $\left({ }^{*} p<0.05\right)$ than that of the predicted responses $(S=$ 0.98). Values are mean $\pm S E$. In both $A$ and $\boldsymbol{B}$, the selectivity of the population spike output (gray circles) and the synaptic response amplitude (triangles) are shown (data from Fig. 3). cell in Figure 6, that an increased number of spikes per suprathreshold trial did not confound the results. In summary, for rat barrel cortex neurons, an increase in spike threshold at nonpreferred directions enhances the selectivity of whisker-evoked responses by effectively decreasing the probability that a nonpreferred stimulus will evoke a suprathreshold response. In addition, the increase in threshold is coincident with a decrease in the rate of membrane depolarization, which is, at least in part, directly regulating threshold.

\section{Discussion}

Understanding the mechanisms underlying the tuning for stimulus features of single cortical neurons is critical to understanding information representation in cortical circuits. The feature selectivity of the spike output of a neuron may result solely from the combination of the tuning characteristics of its synaptic inputs, or it may be enhanced by postsynaptic mechanisms. Our results show that the tuning of the spike output of barrel neurons to the angular direction of whisker deflection is sharper than the synaptic input. As in other sensory cortices (Carandini and Ferster, 2000; Volgushev et al., 2000; Wehr and Zador, 2003; Zhang et al., 2003), this effect results from the rectifying nonlinearity imposed by the spike threshold in the input-output relationship of the cell. In addition, our results show that spike threshold is stimulus dependent and increases as the direction of deflection diverges from the PD. The systematic increase in threshold is correlated with a decrease in the $d V_{\mathrm{m}} / d t$ of the synaptic response. Finally, by comparing the tuning properties of the measured spike output to the tuning properties predicted by applying a fixed threshold to the synaptic responses, we show for the first time that the systematic direction-specific changes in spike threshold significantly enhance the direction selectivity of barrel cortex neurons.

The rise in spike threshold, concomitant with the decrease in $d V_{\mathrm{m}} / d t$, is probably caused by an increased proportion of $\mathrm{Na}^{+}$channels entering the inactivated state during the slower depolarization (Hodgkin and Huxley, 1952; Noble, 1966; Traub and Miles, 1991; Fleidervish et al., 1996; Martina and Jonas, 1997). An increased proportion of inactivated channels increases the level of depolarization needed to generate a net inward membrane current with negative slope conductance, which determines threshold (Hodgkin and Huxley, 1952; Noble and 
Stein, 1966). Given that the difference in latency between spikes initiated at the highest and lowest threshold is $<5 \mathrm{~ms}$, and all spikes were initiated below $-50 \mathrm{mV}$, it is unlikely that the activation of the delayed rectifier potassium current plays an important role in increasing threshold (Traub and Miles, 1991; Martina et al., 1998).

Other factors, such as the change input conductance caused by the synaptic response itself, may also affect threshold. However, an increase in a shunting conductance that would cause an increase in threshold would also result in a steeper slope of the synaptic response, which is the opposite of what we observe. Intrinsic voltage-dependent conductances, located either in the soma (Axmacher and Miles, 2004) or in the dendrites (Hausser et al., 2000; Reyes, 2001), may play an important role in determining the rate of depolarization immediately preceding the action potential thereby affecting threshold. However, the selectivity of neurons recorded with pipettes containing $N$-ethyl bromide quaternary salt (QX-314), which blocks several voltage-gated currents including potassium and calcium (Connors and Prince, 1982; Mulle et al., 1985), was comparable with that measured in control conditions (data not shown). Furthermore, for the cells recorded with QX-314, the selectivity of the synaptic response amplitude was also higher at depolarized baseline $V_{\mathrm{m}}$ s near spike threshold (as in the control conditions). This indicates that the intrinsic currents possibly activated by the depolarizing current do not play a substantial role in determining the direction dependent differences in rate of rise of the synaptic response.

Other factors determining the rate of rise of the synaptic response include concomitant inhibitory input (Wehr and Zador, 2003; Wilent and Contreras, 2004), the degree of synchronization of the excitatory input, and the location of synapses on the somatodendritic membrane. Inhibitory input may simply add linearly to the excitatory input and decrease the slope of the synaptic response, or it may shunt excitatory input by increasing membrane conductance without necessarily causing hyperpolarization (Borg-Graham et al., 1998; Hirsch et al., 1998). The degree of synchronization of thalamocortical input has been shown previously to play a key role for the encoding of velocity/acceleration in layer 4 of barrel cortex (Pinto et al., 2000, 2003), which results in an increase in $d V_{\mathrm{m}} / d t$ of the synaptic response (Wilent and Contreras, 2004). A similar role for thalamocortical input synchronization in the encoding of direction has been suggested by the increase in the amplitude of local field potentials recorded in thalamic barreloids to preferred versus nonpreferred directions (Temereanca and Simons, 2003) and by the dependence of angular tuning on deflection velocity (Lee and Simons, 2004). We cannot determine whether decreases in input synchronization underlie the decrease in $d V_{\mathrm{m}} / d t$ associated with nonpreferred directions. However, the relationship between spike threshold and $d V_{\mathrm{m}} / d t$ increases the sensitivity of barrel cortex neurons to rapid depolarizations and therefore to synchronized input. This enhances the signal-to-noise ratio of the system by limiting the ability of poorly temporally correlated inputs, which depolarize the cell more slowly, to evoke spikes. Finally, input location may also be a critical factor, particularly in layer 4 , where it has been demonstrated recently that minicolumns exist in which thalamocortical axonal arbors that share a common direction preference are colocalized (Bruno et al., 2003).

An important functional consequence of the sharpening of direction tuning by the spike threshold is that it reduces the requirements for finely tuned synaptic input. Direction selectivity is already encoded in the response of trigeminal neurons (Lichtenstein et al., 1990; Minnery and Simons, 2003). In ventrobasal thalamus, thalamocortical cells with like angular preference are clustered within the barreloids (Timofeeva et al., 2003). In cortex, direction selectivity is determined in part by the converging pattern of thalamic afferents with similar direction preference to barrel (layer 4) neurons (Bruno et al., 2003). Furthermore, thalamic convergence results in the arrangement of similar direction preferences in vertical domains within barrels (Bruno et al., 2003). However, the degree of convergence and divergence of thalamic input (Jones, 2001) and the large amount of recurrent connectivity in cortex (Douglas et al., 1995) do not support a fine-tuned anatomical organization that could explain the physiological findings and opens the question of how such domains are organized. Reconciling our results with the anatomy requires elucidating how the dynamics of the inputs vary with respect to direction.

Finally, a variable spike threshold may also influence spike time reliability. For less steep depolarizations, the concurrent increase in threshold increases the time window in which a spike may occur. The rate of depolarization is influenced by the balance of excitation and inhibition, which is a strong determinant of spike time reliability (Pouille and Scanziani, 2001; Wehr and Zador, 2003).

We have established that as the direction of deflection deviates from the PD, threshold systematically increases and the rate of membrane depolarization systematically decreases. We also demonstrated that the increase in action potential threshold enhances the direction selectivity of barrel neurons to the direction of deflection. Given that the intrinsic regulation of spike threshold by preceding $V_{\mathrm{m}}$ is a ubiquitous biophysical property of neurons, we propose that the regulation of spike threshold by the stimulus-dependent rate of membrane depolarization may be a general mechanism used by the neocortex to enhance neuronal feature selectivity.

\section{References}

Alonso JM, Usrey WM, Reid RC (1996) Precisely correlated firing in cells of the lateral geniculate nucleus. Nature 383:815-819.

Axmacher N, Miles R (2004) Intrinsic cellular currents and the temporal precision of EPSP-action potential coupling in CA1 pyramidal cells. J Physiol (Lond) 555:713-725.

Azouz R, Gray CM (2000) Dynamic spike threshold reveals a mechanism for synaptic coincidence detection in cortical neurons in vivo. Proc Natl Acad Sci USA 97:8110-8115.

Azouz R, Gray CM (2003) Adaptive coincidence detection and dynamic gain control in visual cortical neurons in vivo. Neuron 37:513-523.

Bonds AB (1989) Role of inhibition in the specification of orientation selectivity of cells in the cat striate cortex. Vis Neurosci 2:41-55.

Borg-Graham LJ, Monier C, Fregnac Y (1998) Visual input evokes transient and strong shunting inhibition in visual cortical neurons. Nature 393:369-373.

Brecht M, Sakmann B (2002) Dynamic representation of whisker deflection by synaptic potentials in spiny stellate and pyramidal cells in the barrels and septa of layer 4 rat somatosensory cortex. J Physiol (Lond) 543:49-70.

Brecht M, Roth A, Sakmann B (2003) Dynamic receptive fields of reconstructed pyramidal cells in layers 3 and 2 of rat somatosensory barrel cortex. J Physiol (Lond) 553:243-265.

Bruno RM, Simons DJ (2002) Feedforward mechanisms of excitatory and inhibitory cortical receptive fields. J Neurosci 22:10966-10975.

Bruno RM, Khatri V, Land PW, Simons DJ (2003) Thalamocortical angular tuning domains within individual barrels of rat somatosensory cortex. J Neurosci 23:9565-9574.

Bryant HL, Segundo JP (1976) Spike initiation by transmembrane current: a white-noise analysis. J Physiol (Lond) 260:279-314.

Carandini M, Ferster D (2000) Membrane potential and firing rate in cat primary visual cortex. J Neurosci 20:470-484.

Chance FS, Nelson SB, Abbott LF (1999) Complex cells as cortically amplified simple cells. Nat Neurosci 2:277-282. 
Connors BW, Gutnick MJ (1990) Intrinsic firing patterns of diverse neocortical neurons. Trends Neurosci 13:99-104.

Connors BW, Prince DA (1982) Effects of local anesthetic QX-314 on the membrane properties of hippocampal pyramidal neurons. J Pharmacol Exp Ther 220:476-481.

Douglas RJ, Koch C, Mahowald M, Martin KA, Suarez HH (1995) Recurrent excitation in neocortical circuits. Science 269:981-985.

Ferster D (2004) Assembly of receptive fields in primary visual cortex. In: The visual neurosciences (Werner JS, Chalupa LM, eds), pp 695-703. Cambridge, MA: MIT.

Fleidervish IA, Friedman A, Gutnick MJ (1996) Slow inactivation of Na+ current and slow cumulative spike adaptation in mouse and guinea-pig neocortical neurones in slices. J Physiol (Lond) 493:83-97.

Fricker D, Verheugen JA, Miles R (1999) Cell-attached measurements of the firing threshold of rat hippocampal neurones. J Physiol (Lond) 517:791-804.

Granit R, Kernell D, Shortess GK (1963) Quantitative aspects of repetitive firing of mammalian motoneurones, caused by injected currents. J Physiol (Lond) 168:911-931.

Hausser M, Spruston N, Stuart GJ (2000) Diversity and dynamics of dendritic signaling. Science 290:739-744.

Henze DA, Buzsaki G (2001) Action potential threshold of hippocampal pyramidal cells in vivo is increased by recent spiking activity. Neuroscience 105:121-130.

Higley MJ, Contreras D (2003) Nonlinear integration of sensory responses in the rat barrel cortex: an intracellular study in vivo. J Neurosci 23:10190-10200.

Hirsch JA, Alonso JM, Reid RC, Martinez LM (1998) Synaptic integration in striate cortical simple cells. J Neurosci 18:9517-9528.

Hodgkin AL, Huxley AF (1952) A quantitative description of membrane current and its application to conduction and excitation in nerve. J Physiol (Lond) 117:500-544.

Hubel DH, Wiesel TN (1962) Receptive fields, binocular interaction and functional architecture in the cat's visual cortex. J Physiol (Lond) 160:106-154.

Jones EG (2001) The thalamic matrix and thalamocortical synchrony. Trends Neurosci 24:595-601.

Lee SH, Simons DJ (2004) Angular tuning and velocity sensitivity in different neuron classes within layer 4 of rat barrel cortex. J Neurophysiol 91:223-229.

Lichtenstein SH, Carvell GE, Simons DJ (1990) Responses of rat trigeminal ganglion neurons to movements of vibrissae in different directions. Somatosens Mot Res 7:47-65.

Martin KA (2002) Microcircuits in visual cortex. Curr Opin Neurobiol $12: 418-425$.

Martina M, Jonas P (1997) Functional differences in $\mathrm{Na}+$ channel gating between fast-spiking interneurones and principal neurones of rat hippocampus. J Physiol (Lond) 505:593-603.

Martina M, Schultz JH, Ehmke H, Monyer H, Jonas P (1998) Functional and molecular differences between voltage-gated $\mathrm{K}^{+}$channels of fastspiking interneurons and pyramidal neurons of rat hippocampus. J Neurosci 18:8111-8125.

Minnery BS, Simons DJ (2003) Response properties of whisker-associated trigeminothalamic neurons in rat nucleus principalis. J Neurophysiol 89:40-56.

Moore CI, Nelson SB (1998) Spatio-temporal subthreshold receptive fields in the vibrissa representation of rat primary somatosensory cortex. J Neurophysiol 80:2882-2892.

Moore CI, Nelson SB, Sur M (1999) Dynamics of neuronal processing in rat somatosensory cortex. Trends Neurosci 22:513-520.
Movshon JA, Thompson ID, Tolhurst DJ (1978) Receptive field organization of complex cells in the cat's striate cortex. J Physiol (Lond) 283:79-99.

Mulle C, Steriade M, Deschenes M (1985) The effects of QX314 on thalamic neurons. Brain Res 333:350-354.

Nelson S, Toth L, Sheth B, Sur M (1994) Orientation selectivity of cortical neurons during intracellular blockade of inhibition. Science 265:774-777.

Noble D (1966) Applications of Hodgkin-Huxley equations to excitable tissues. Physiol Rev 46:1-50.

Noble D, Stein RB (1966) The threshold conditions for initiation of action potentials by excitable cells. J Physiol (Lond) 187:129-162.

Nunez A, Amzica F, Steriade M (1993) Electrophysiology of cat association cortical cells in vivo: intrinsic properties and synaptic responses. J Neurophysiol 70:418-430.

Pinto DJ, Brumberg JC, Simons DJ (2000) Circuit dynamics and coding strategies in rodent somatosensory cortex. J Neurophysiol 83:1158-1166.

Pinto DJ, Hartings JA, Brumberg JC, Simons DJ (2003) Cortical damping: analysis of thalamocortical response transformations in rodent barrel cortex. Cereb Cortex 13:33-44.

Pouille F, Scanziani M (2001) Enforcement of temporal fidelity in pyramidal cells by somatic feed-forward inhibition. Science 293:1159-1163.

Reyes A (2001) Influence of dendritic conductances on the input-output properties of neurons. Annu Rev Neurosci 24:653-675.

Schlue WR, Richter DW, Mauritz KH, Nacimiento AC (1974) Responses of cat spinal motoneuron somata and axons to linearly rising currents. J Neurophysiol 37:303-309.

Shoykhet M, Shetty P, Minnery BS, Simons DJ (2003) Protracted development of responses to whisker deflection in rat trigeminal ganglion neurons. J Neurophysiol 90:1432-1437.

Simons DJ (1983) Multi-whisker stimulation and its effects on vibrissa units in rat SmI barrel cortex. Brain Res 276:178-182.

Simons DJ, Carvell GE (1989) Thalamocortical response transformation in the rat vibrissa/barrel system. J Neurophysiol 61:311-330.

Temereanca S, Simons DJ (2003) Local field potentials and the encoding of whisker deflections by population firing synchrony in thalamic barreloids. J Neurophysiol 89:2137-2145.

Timofeeva E, Merette C, Emond C, Lavallee P, Deschenes M (2003) A map of angular tuning preference in thalamic barreloids. J Neurosci 23:10717-10723.

Traub RD, Miles R (1991) Neuronal networks of the hippocampus. New York: Cambridge UP.

Volgushev M, Pernberg J, Eysel UT (2000) Comparison of the selectivity of postsynaptic potentials and spike responses in cat visual cortex. Eur J Neurosci 12:257-263.

Volgushev M, Pernberg J, Eysel UT (2002) A novel mechanism of response selectivity of neurons in cat visual cortex. J Physiol (Lond) 540:307-320.

Wehr M, Zador AM (2003) Balanced inhibition underlies tuning and sharpens spike timing in auditory cortex. Nature 426:442-446.

Wilent WB, Contreras D (2004) Synaptic responses to whisker deflections in rat barrel cortex as a function of cortical layer and stimulus intensity. J Neurosci 24:3985-3998.

Zhang LI, Tan AY, Schreiner CE, Merzenich MM (2003) Topography and synaptic shaping of direction selectivity in primary auditory cortex. Nature 424:201-205.

Zhu JJ, Connors BW (1999) Intrinsic firing patterns and whisker-evoked synaptic responses of neurons in the rat barrel cortex. J Neurophysiol 81:1171-1183. 